\title{
Claudin-4 expression is associated with tumor invasion, MMP-2 and MMP-9 expression in gastric cancer
}

\author{
TSANN-LONG HWANG ${ }^{1}$, LI-YU LEE ${ }^{2}$, CHEE-CHAN WANG ${ }^{3}$, \\ YING LIANG $^{4}$, SHU-FANG HUANG ${ }^{1}$ and CHI-MING WU ${ }^{3}$
}

\begin{abstract}
Departments of ${ }^{1}$ Surgery, and ${ }^{2}$ Pathology, Chang Gung Memorial Hospital; ${ }^{3}$ Department of Cosmetic Science, Vanung University; ${ }^{4}$ Graduate Institute of Basic Medical Science, Chang Gung University, Tao-Yuan, Taiwan, R.O.C.
\end{abstract}

Received April 28, 2010; Accepted June 28, 2010

DOI: $10.3892 /$ etm.2010.116

\begin{abstract}
Claudin-4 is a member of the claudin family, a large family of transmembrane proteins that are essential in the formation and maintenance of tight junctions. Matrix metalloproteinase (MMP)-2 and -9 degrade type IV collagen of the extracellular matrix and basal membranes. Claudin-4 activates MMP-2, indicating that claudin-mediated increased cancer cell invasion may result from the activation of MMP proteins. In the present study, we used immunohistochemistry to examine the expression levels of claudin-4, MMP-2 and MMP-9 in 189 gastric cancer samples, and analyzed their correlation with tumor invasion, clinicopathologic parameters and clinical outcome. The relationship between claudin-4 expression and MMP-2 and -9 expression was also investigated. The expression of claudin-4 was found to be significantly higher in gastric cancer cases with advanced depth of wall invasion, lymph node metastasis, lymphatic invasion and higher TNM stage. Further analysis revealed claudin-4 expression to be significantly correlated with the expression of MMP-2 and -9. Kaplan-Meier survival analysis indicated that MMP-9 expression was correlated with poor prognosis. These results suggest that claudin-4 expression is associated with tumor invasion and with MMP-2 and -9 expression in gastric cancer. Additionally, MMP-9 expression was demonstrated to serve as a prognostic factor in patients with gastric cancer.
\end{abstract}

\section{Introduction}

Claudins are tight junctional proteins which are present at the epithelial and endothelial cell membranes $(1,2)$, and are the major integral membrane proteins forming the backbone of tight junctions. Tight junctions form the primary barrier to the paracellular transport of solutes across cells, and also play

Correspondence to: Dr Chi-Ming Wu, Department of Cosmetic Science, Vanung University, No. 1 Van-Nung Rd., Tao-Yuan 320, Taiwan, R.O.C.

E-mail: chimingwu@mail.vnu.edu.tw

Key words: claudin-4, matrix metalloproteinase-2, matrix metalloproteinase-9, gastric cancer a critical role in establishing and maintaining epithelial cell polarity $(3,4)$. The claudin family consists of 23 transmembrane proteins exhibiting distinct tissue- and development-specific distribution patterns (5). The carboxylic terminal region of claudin proteins contains a PDZ domain-binding motif that potentially interacts with a number of PDZ domain-containing proteins, such as $\mathrm{ZO}$ proteins $(6,7)$. These interactions also serve as adapters for other proteins involved in cell signaling. A number of other cytosolic and nuclear proteins, including regulatory proteins such as Rab3b, tumor suppressors such as PTEN and transcription factors such as ZONAB, also interact directly or indirectly with the tight junction complex (8-10). These interactions suggest that tight junctions, in addition to acting as barriers to the paracellular flow of solutes, may play an important role in regulating other cell functions, such as proliferation and tumor suppression.

Modulations in tight junction structure and function have been observed in epithelial tumorigenesis $(11,12)$. A tissue microarray study showed that claudin-1, -3 and -4 are strongly expressed in most cases of intestinal-type gastric cancer, but are less frequently expressed in diffuse-type gastric cancer (13). Using cDNA microarray and immunohistochemical analysis, our group previously showed that the expression of claudin-4 was significantly higher in intestinal-type than in diffuse-type gastric cancer $(14,15)$. Other studies have shown that claudin-2 expression gradually increases during the multistage process of gastric carcinogenesis $(16,17)$. In addition, several studies have found aberrant claudin expression in various types of cancer, including increased expression of claudin-3 and -4 in prostate and uterine cancers $(18,19)$, high claudin-4 expression in pancreatic cancer (20), down-regulation of claudin-7 in head and neck cancer (21) and metastatic breast cancer (22), and an increase in claudin-3 and -4 in breast cancer (23). However, the exact role of claudin overexpression and the functional importance of these proteins in the development of gastric cancer remain unclear.

Gastric cancer is one of the most common malignant tumors of the alimentary tract. At the time of diagnosis, it usually shows extensive local tumor invasion and frequent spread to metastatic sites, particularly the lymph nodes. It is thus characterized by late clinical presentation, rapid progression and a poor survival prognosis (24). The spread of malignant tumors is a multistep process, and many of the stages of tumor 
invasion require degradation or breakdown of the extracellular matrix and connective tissue surrounding tumor cells $(25,26)$. The matrix metalloproteinases (MMPs) are a family of zinccontaining enzymes involved in the degradation of different components of the extracellular matrix. There is considerable evidence indicating that individual MMPs play crucial roles in tumor invasion and tumor spread (27-32). Some studies have suggested a major role for MMP-2 and - 9 in the digestion of basement membrane type IV collagen as an important mechanism for vessal invasion and metastasis in gastric cancer $(33,34)$.

Recent studies have indicated the modulatory effects of claudins on MMP activation. Agarwal et al showed that claudin-3 and -4 expression in ovarian epithelial cells enhanced invasion and was associated with increased MMP-2 activity (35). Oku et al showed that claudin-1 enhanced the invasive activity of oral squamous cell carcinoma cells by promoting the cleavage of the laminin-5 $\gamma 2$ chain via MMP-2 and membrane-type MMP-1 (36). Takehara et al revealed that the overexpression of claudin-4 specifically stimulated the invasive activity of colonic cancer cells and increased MMP-2 and -9 activity (37). In the present study, we examined the expression levels of claudin-4, MMP-2 and -9 in gastric cancer in order to analyze their correlation with tumor invasion, clinicopathologic parameters and clinical outcome of gastric cancer patients. We also investigated the relationship between claudin-4 expression and MMP-2 and -9 expression in gastric cancer.

\section{Materials and methods}

Patients and specimens. A consecutive series of 189 tissue specimens was collected from patients with gastric cancer who underwent subtotal or total resection by gastrectomy at Chang Gung Memorial Hospital (CGMH), Taiwan between January 2001 and December 2002. Written informed consent was obtained before sample collection, and the study was approved by the Institutional Review Board of CGMH. The patients comprised 110 males and 79 females with a mean age of 62 years (range 24-90). The age and gender of the patients, tumor location, tumor size, cell differentiation, depth of wall invasion, status of lymph node metastasis, vascular invasion, lymphatic invasion and desmoplastic reaction were obtained from histopathology records. The stage of gastric cancer was described according to the 1997 tumor-node-metastasis (TNM) classification of malignant tumors of the American Joint Committee on Cancer. Follow-up was conducted until December 2007, for a minimum follow-up time of 5 years. The tissue specimens were formalin-fixed and paraffin-embedded, then stained with $\mathrm{H} \& \mathrm{E}$ and classified by a pathologist. The results were compared to histopathology records from CGMH. Final pathology was determined by consensus, with review if necessary.

Immunohistochemistry. Tissue blocks were constructed according to the method of Schraml et al (38), and the most representative morphological areas of the tumors were used in the study. The specimen sections were deparaffinized, treated with $3 \%$ hydrogen peroxide and microwaved after pre-treatment in $10 \mathrm{mM}$ citric acid to retrieve antigenicity. The sections were incubated with blocking solution containing PBS and $1 \%$ bovine serum albumin for $20 \mathrm{~min}$ at room temperature, and then incubated overnight at $4^{\circ} \mathrm{C}$ with an anti-claudin- 4 antibody (1:100; Zymed, San Francisco, CA, USA), an anti-MMP-2 monoclonal antibody (1:50; Lab Vision Corporation, Fremont, CA, USA) and an anti-MMP-9 monoclonal antibody (1:50; Lab Vision Corporation), respectively. After washing 4 times with Tris-buffered saline, the sections were incubated with biotinylated secondary antibody (Santa Cruz Biotechnology, Santa Cruz, CA, USA). The immunocomplex was visualized by the immonoglobulin enzyme bridge technique using the Dako LSAB 2 System, HRP kit (Dako Corp., Carpinteria, CA, USA) with 3,3'diaminobenzidine tetrachloride as a substrate. The sections were counterstained with hematoxylin, dehydrated with graded alcohol, cleared with xylene and mounted with coverslips.

Scoring of immunostaining. The results of immunostaining were scored according to a previous report (39) as follows: the immunostaining reaction was evaluated by subjective assessments of the median staining intensity $(0$, no stain; 1 , weak; 2 , moderate; 3 , strong stain) and by the fraction of stained cells in percentage categories $(0,0-9 \% ; 1,10-49 \% ; 2,50-89 \%$; and $3, \geq 90 \%$ ). This scoring system was previously shown to be reproducible (40). Scores of 0-3 were determined as follows: percentage categories and staining were each ranked as indicated above. The ranks for percentage and staining intensity were multiplied by each other, divided by 3 and rounded up to the nearest whole number (40). The results of immunostaining were classified as negative (whole number 0 ) or positive (whole number 1-3), respectively (Fig. 1).

Statistical analysis. The $\chi^{2}$ test or Fisher's exact test were used to test for an association between claudin-4, MMP-2 and -9 expression and the clinicopathologic parameters of the patients. Disease-free survival was defined as the time from surgery to the first relapse of cancer, occurence of a second primary tumor or death of any cause. Univariate survival analysis was assessed by the Kaplan-Meier method, and the significance of differences between groups was analyzed using the log rank test or the log rank test for trend. Stepwise multivariate survival analysis was performed according to the Cox proportional hazards model. All reported P-values were two-sided, and P-values $<0.05$ were considered significant.

\section{Results}

Claudin-4, MMP-2 and MMP-9 expression in gastric cancer. Claudin-4 was expressed in the membrane of gastric adenocarcinoma cells in $84.7 \%(160 / 189)$ of cases. MMP-2 and -9 were expressed in the cytoplasm of gastric adenocarcinoma cells in $81 \%(153 / 189)$ and $89.4 \%(169 / 189)$ of the cases, respectively (Fig. 1).

Claudin-4, MMP-2 and MMP-9 expression in relation to clinicopathologic parameters. The expression of claudin-4 was significantly higher in males than in females $(\mathrm{P}=0.046)$, and was positively correlated with tumor size $(\mathrm{P}=0.008)$ and desmoplastic reaction $(\mathrm{P}=0.027)$. The expression of claudin-4 was significantly higher in gastric cancer with advanced depth of wall invasion $(\mathrm{P}=0.008)$, lymph node metastasis $(\mathrm{P}=0.005)$, 

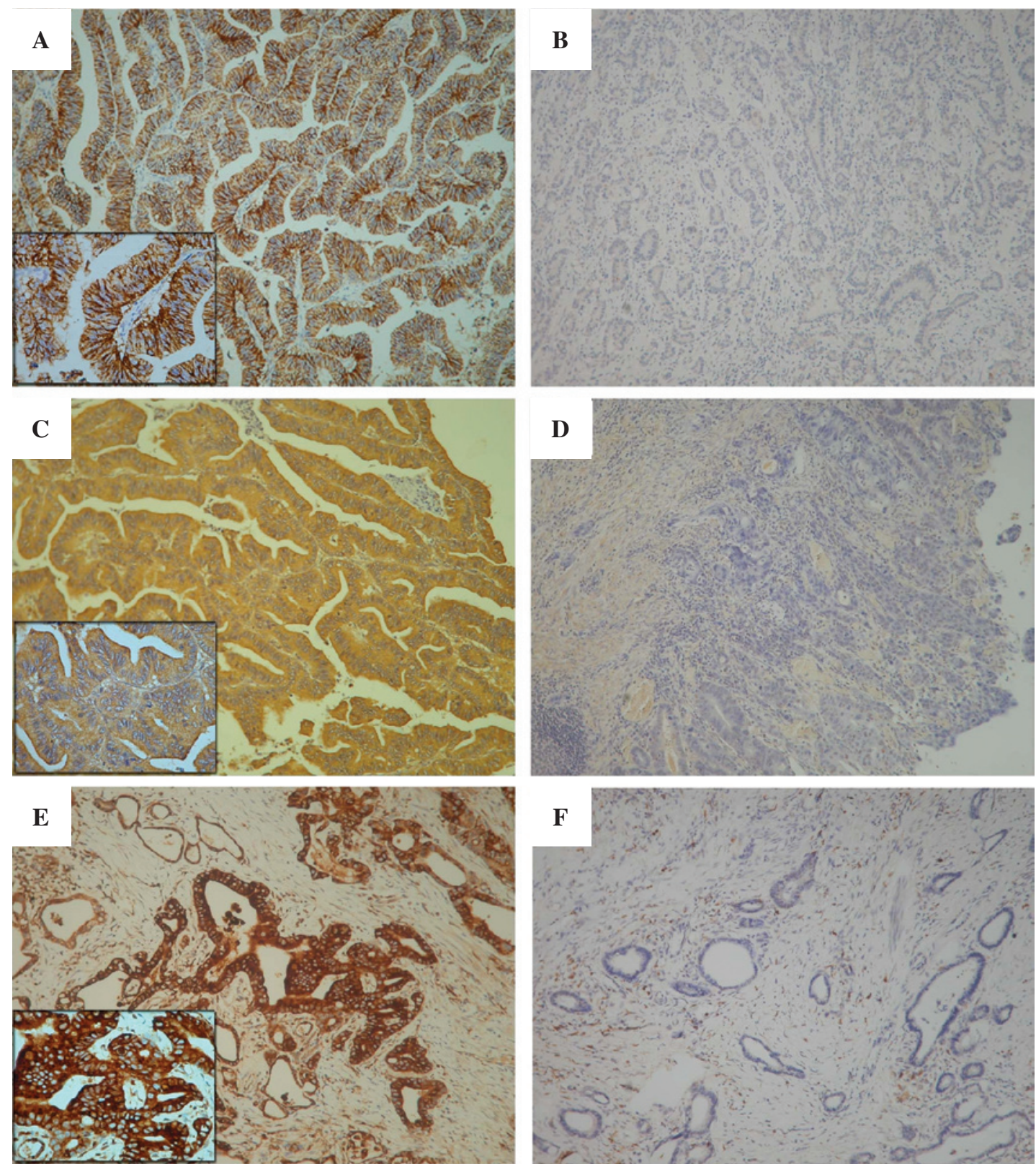

Figure 1. Immunohistochemistry of claudin-4, MMP-2 and MMP-9 in representative samples of gastric cancer. (A) Positive staining for claudin-4 in the cell membrane of gastric cancer cells. (B) Negative staining for claudin-4 in a gastric cancer specimen. (C) Positive staining for MMP-2 in the cytoplasm of gastric cancer cells. (D) Negative staining for MMP-2 in a gastric cancer specimen. (E) Positive staining for MMP-9 in the cytoplasm of gastric cancer cells. (F) Negative staining for MMP-9 in a gastric cancer specimen. Magnification x100. Inset shows immunohistochemistry at a high magnification (x400).

lymphatic invasion $(\mathrm{P}=0.001)$ and high TNM stage $(\mathrm{P}=0.004)$, but was not correlated with age, tumor location, cell differentiation or vascular invasion (Table I).

The expression of MMP-2 was significantly higher in males than in females $(\mathrm{P}=0.036)$, and was significantly correlated with tumor location $(\mathrm{P}=0.01)$, tumor size $(\mathrm{P}=0.024)$, cell differentiation $(\mathrm{P}=0.04)$ and desmoplastic reaction $(\mathrm{P}=0.021)$, but not with age, depth of wall invasion, lymph node metastasis, vascular invasion, lymphatic invasion or TNM stage (Table I).

MMP-9 expression was positively correlated with tumor size $(\mathrm{P}=0.004)$ and desmoplastic reaction $(\mathrm{P}=0.02)$. As with claudin-4, the expression of MMP-9 was significantly higher in gastric cancer with advanced depth of wall invasion ( $\mathrm{P}=0.002)$, lymph node metastasis $(\mathrm{P}=0.038)$, lymphatic inva- sion $(\mathrm{P}=0.046)$ and higher TNM stage $(\mathrm{P}=0.008)$, but was not correlated with age, gender, tumor location, cell differentiation or vascular invasion (Table I).

Correlation of claudin- 4 expression with MMP-2 and MMP-9 expression. Further analysis of the relationship between claudin-4 expression and MMP-2 and -9 expression revealed claudin-4 expression to be significantly correlated with the expression of these two proteins $(\mathrm{P}=0.005$ and 0.018 , respectively; Table II). To better define the pattern of co-expression between claudin- 4 and these two proteins, immunostaining was conducted in serial sections of gastric cancer. Of 189 specimens, $135(71.4 \%)$ were positive for claudin-4- and MMP-2, and 147 (77.8\%) were positive for claudin-4- and MMP-9 (Table II and Fig. 2). 


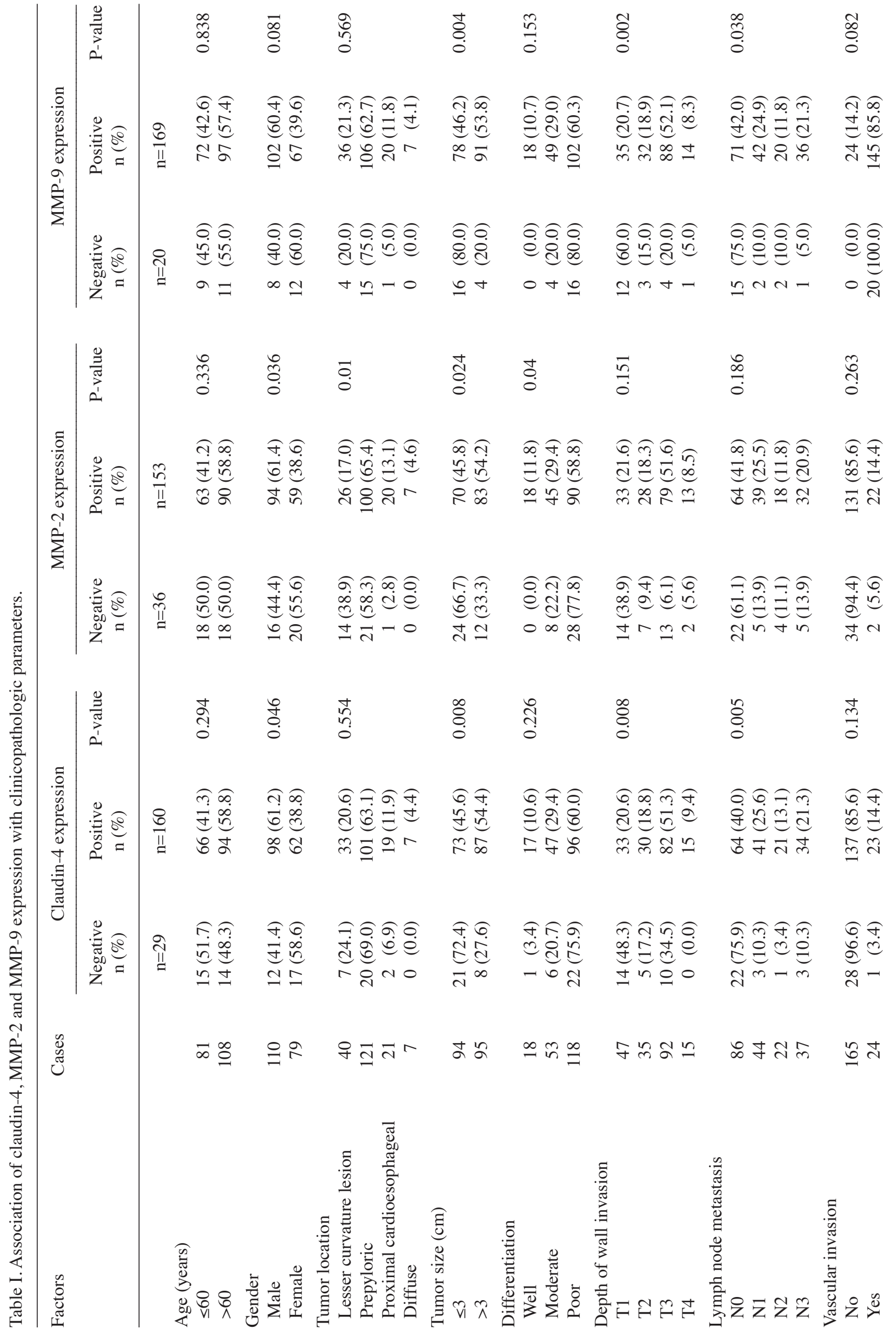




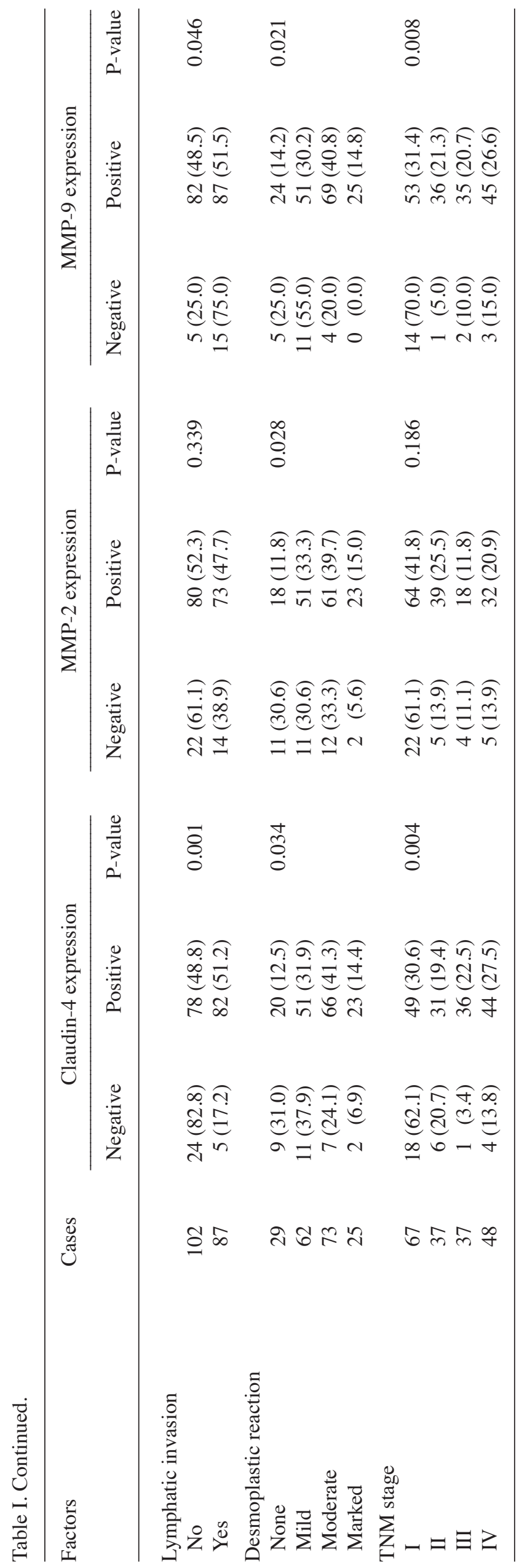

Table II. Association of claudin-4 expression with MMP-2 and MMP-9 expression.

\begin{tabular}{lccc}
\hline & \multicolumn{3}{c}{ Claudin-4 expression } \\
\cline { 2 - 4 } Factors & $\begin{array}{c}\text { Negative } \\
\mathrm{n}(\%)\end{array}$ & $\begin{array}{c}\text { Positive } \\
\mathrm{n}(\%)\end{array}$ & P-value \\
\hline & $\mathrm{n}=29$ & $\mathrm{n}=160$ & \\
MMP-2 expression & & & 0.005 \\
Negative (-) & $11(37.9)$ & $25(15.6)$ & \\
Positive (+) & $18(62.1)$ & $135(84.4)$ & \\
MMP-9 expression & & & 0.018 \\
Negative (-) & $7(24.1)$ & $13(8.1)$ & \\
Positive (+) & $22(75.9)$ & $147(91.9)$ & \\
\hline
\end{tabular}

Prognostic implications of claudin-4, MMP-2 and MMP-9 expression in gastric cancer. MMP-9 expression was correlated with a poor prognosis $(\mathrm{P}=0.041$; Table III and Fig. $3 \mathrm{C})$. Neither claudin-4 nor MMP-2 expression was correlated with survival (Table III, Fig. 3A and B). Other prognostic factors were type of gastrectomy, tumor location, large tumor size, poor cell differentiation, advanced penetration depth, presence of nodal metastases, presence of vascular or lymphatic invasion, marked desmoplastic reaction and higher stage. In multivariate analysis, depth of invasion, lymph node metastasis and lymphatic invasion were independent prognostic factors (Table IV).

\section{Discussion}

In this study, claudin-4, MMP-2 and MMP-9 expression was examined in 189 cases of gastric cancer, and was associated with patient clinicopathologic factors. Claudin-4 expression was correlated with depth of wall invasion, lymph node metastasis and lymphatic invasion, and was significantly correlated with MMP-2 and -9 expression. These results are consistent with those obtained in a cancer cell model $(35,37)$. Agarwal et al showed that claudin-4 expression in ovarian epithelial cells enhanced cell invasion and was associated with increased MMP-2 activity (35). Takehara et al also showed that the overexpression of claudin- 4 in colonic cancer cells stimulated invasive activity and MMP-2 and -9 activity (37). Although it is generally believed that an alteration in claudin expression is involved in tumorigenesis, the role of claudin-4 in the regulation of cancer-related cell functions, such as invasion, remains unclear. It is known that claudins affect cell physiology by recruiting signal transductionrelated molecules at tight junctions. Claudin-4 affects the expression and activity of MMP-2 and -9 either directly or by modulating signal transduction; consequently, these two proteins stimulate cell invasion.

Recent studies also indicate that the overexpression of claudins is correlated with tumor invasion. Wu et al demonstrated that the overexpression of claudin-1 was correlated with the invasiveness and metastasis of gastric cancer (41). 

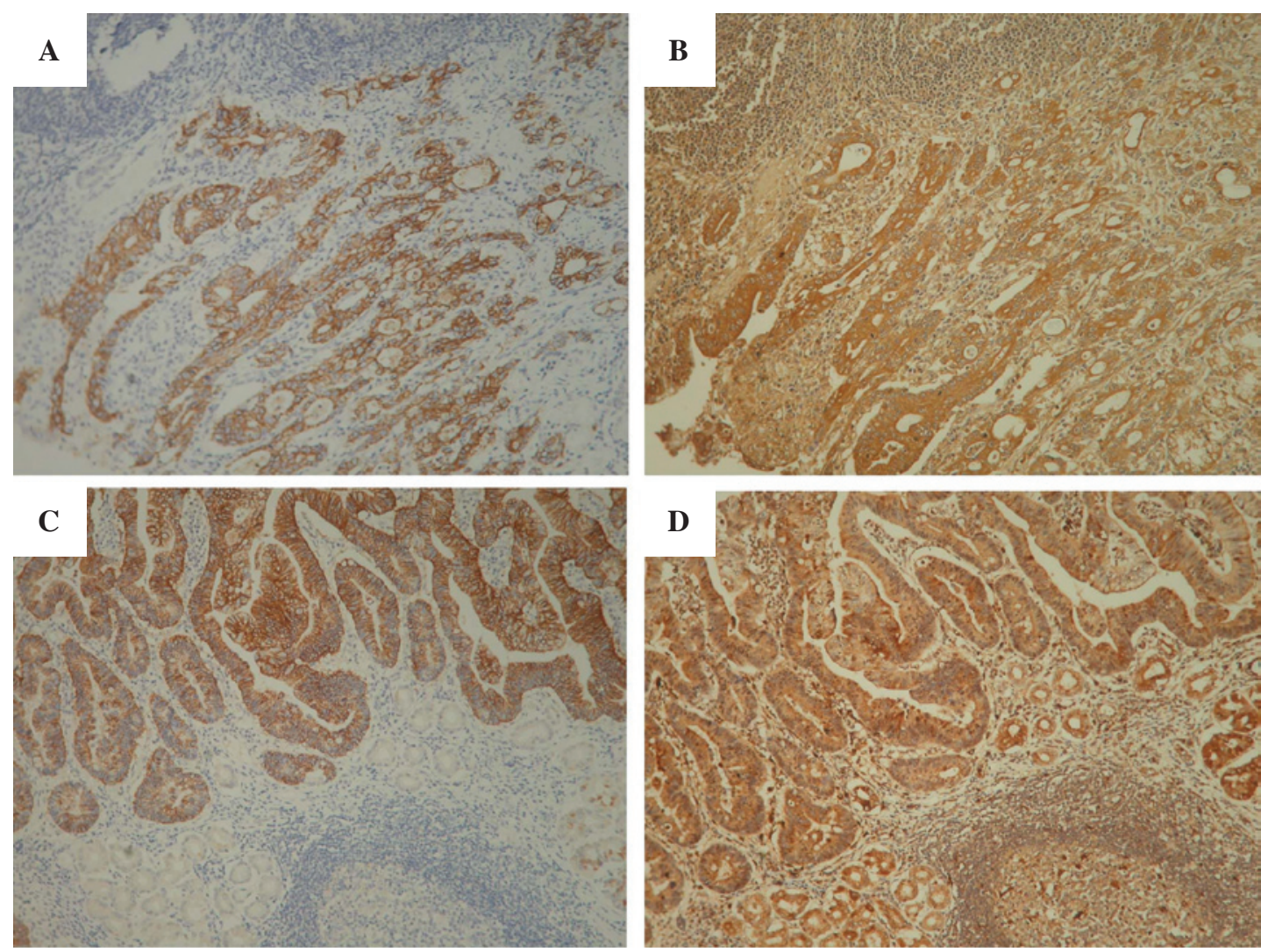

Figure 2. Immunohistochemical analysis using serial sections of gastric cancer showing co-expression of claudin-4 (A), MMP-2 (B), claudin-4 (C) and MMP-9 (D) in gastric cancer. Magnification x100.

Kinugasa et al revealed that the expression of claudin-1 and -2 was up-regulated in colorectal cancer, and that this up-regulation was correlated with the depth of tumor invasion (42). Dhawan et al showed that claudin-1 expression increased with the progression of colon carcinoma and metastasis (43). Nevertheless, some studies have shown that the downregulation of claudins is correlated with tumor invasion. Ueda et al showed that decreased claudin- 4 expression at the invasive front is correlated with cancer invasion and metastasis in colorectal cancer (44). Oshima et al showed that reduced expression of claudin-7 correlated with venous invasion and liver metastasis in colorectal cancer (45). Usami et al reported that reduced expression of claudin-7 at the invasive front of esophageal squamous cell carcinoma may lead to tumor progression and subsequent metastasis (46). Morohashi et al found that decreased expression of claudin-1 correlated with lymphatic node metastasis in breast cancer (47). These reports of decreased claudin protein expression in cancer are consistent with the generally accepted notion that tumorigenesis is accompanied by a disruption of the tight junctions, a process that may play a key role in the loss of cohesion and invasiveness observed in cancer cells.

In gastric cancer, MMP-2 and -9 are linked to tumor invasion and metastasis as well as to a poor prognosis (48-50). Kabashima et al demonstrated a correlation between MMP-9 expression and lymphatic invasion and lymph node positivity in gastric carcinoma (51). The results of Kabashima et al are consistent with the results of the present study, which indicate that MMP-9, but not MMP-2 expression, is positively corre- lated with lymph node metastasis and lymphatic invasion as well as with a poor prognosis. By contrast, certain reports have shown MMP-2 expression to be associated with tumor invasion, lymph node metastasis and survival in gastric cancer (52-54). Allgyer et al demonstrated an association between the immunohistochemical detection of MMP-2 and the prognosis of gastric cancer patients (52). Ji et al showed that the expression of MMP-2 mRNA was significantly correlated with lymph node metastasis and a poor prognosis in gastric cancer patients (53). Monig et al also found that the intensity of MMP-2 staining in tumor cells was significantly correlated with the depth of tumor infiltration, lymph node metastasis and distal metastasis in gastric cancer patients (54). Further studies are required to clearly distinguish the roles and involvement of MMP-2 and -9 in the metastasis of gastric cancer.

In conclusion, claudin-4 expression was correlated with the depth of wall invasion, lymph node metastasis and lymphatic invasion in gastric cancer. Further analysis showed that claudin-4 expression was significantly correlated with MMP-2 and -9 expression. We suggest that claudin-4 affects the expression and activity of MMP-2 and -9 either directly or by modulating signal transduction, and that these two proteins stimulate tumor cell invasion.

\section{Acknowledgements}

This study was supported by a grant from the National Science Council (NSC 98-2314-B-238-001) and a grant from Vanung University, Taiwan (VIT-98-CM-01). 
Table III. Univariate analysis of the clinicopathologic parameters influencing disease-free survival in 189 gastric cancer patients undergoing gastrectomy.

\begin{tabular}{|c|c|c|c|c|c|}
\hline Factors & No. cases & Mean survival (months) & $95 \%$ CI of mean & 5-year survival (\%) & P-value \\
\hline \multicolumn{6}{|l|}{ Age (years) } \\
\hline$\leq 60$ & 81 & 54.6 & $46.9-62.3$ & 58.8 & \multirow[t]{2}{*}{0.7750} \\
\hline$>60$ & 108 & 54.9 & $48.1-61.7$ & 61.2 & \\
\hline \multicolumn{6}{|l|}{ Gender } \\
\hline Male & 110 & 58.5 & $52.0-65.0$ & 65.1 & \multirow[t]{2}{*}{0.0820} \\
\hline Female & 79 & 49.6 & $41.6-57.7$ & 53.3 & \\
\hline \multicolumn{6}{|l|}{ Type of gastrectomy } \\
\hline Total & 42 & 29.3 & $20.2-38.4$ & 33.8 & \multirow[t]{2}{*}{$<0.0001$} \\
\hline Subtotal & 147 & 60.8 & $55.4-66.1$ & 67.7 & \\
\hline \multicolumn{6}{|l|}{ Tumor location } \\
\hline Lesser curvature lesion & 40 & 65.9 & $56.7-75.1$ & 74.5 & \multirow[t]{4}{*}{$<0.0001$} \\
\hline Prepyloric & 121 & 56.7 & $50.4-62.9$ & 62.3 & \\
\hline Proximal cardioesophageal & 21 & 24.8 & $13.2-36.4$ & 30.2 & \\
\hline Diffuse & 7 & 22.9 & $4.5-41.3$ & 28.6 & \\
\hline \multicolumn{6}{|l|}{ Tumor size (cm) } \\
\hline$\leq 3$ & 94 & 72.2 & $67.2-77.1$ & 83.4 & \multirow[t]{2}{*}{$<0.0001$} \\
\hline$>3$ & 95 & 36.9 & $29.5-44.2$ & 36.0 & \\
\hline \multicolumn{6}{|l|}{ Differentiation } \\
\hline Well & 18 & 65.4 & $59.0-71.9$ & 94.4 & \multirow[t]{3}{*}{0.0190} \\
\hline Moderate & 53 & 43.7 & $35.2-52.2$ & 58.1 & \\
\hline Poor & 118 & 52.6 & $46.1-59.0$ & 51.5 & \\
\hline \multicolumn{6}{|l|}{ Depth of invasion } \\
\hline $\mathrm{T} 1$ & 47 & 79.4 & $75.0-83.7$ & 95.7 & \multirow[t]{4}{*}{$<0.0001$} \\
\hline $\mathrm{T} 2$ & 35 & 74.2 & $67.2-81.3$ & 85.4 & \\
\hline $\mathrm{T} 3$ & 92 & 39.3 & $32.1-48.5$ & 38.9 & \\
\hline $\mathrm{T} 4$ & 15 & 8.2 & 4.6-11.7 & 0.0 & \\
\hline \multicolumn{6}{|l|}{ Lymph node metastasis } \\
\hline No & 86 & 74.9 & $70.6-79.3$ & 86.6 & \multirow[t]{4}{*}{$<0.0001$} \\
\hline $\mathrm{N} 1$ & 44 & 60.2 & $50.0-70.4$ & 69.6 & \\
\hline $\mathrm{N} 2$ & 22 & 27.2 & $16.1-38.3$ & 26.0 & \\
\hline N3 & 37 & 11.2 & $7.0-15.4$ & 0.0 & \\
\hline \multicolumn{6}{|l|}{ Vascular invasion } \\
\hline No & 165 & 60.9 & $55.9-66.0$ & 68.1 & \multirow[t]{2}{*}{$<0.0001$} \\
\hline Yes & 24 & 11.1 & $5.4-16.9$ & 4.5 & \\
\hline \multicolumn{6}{|l|}{ Lymphatic invasion } \\
\hline No & 102 & 71.3 & $66.5-76.2$ & 81.5 & $<0.0001$ \\
\hline Yes & 87 & 34.7 & $27.1-42.4$ & 34.3 & \\
\hline Desmoplastic reaction & & & & & \\
\hline None & 29 & 67.4 & $56.5-78.2$ & 79.3 & $<0.0001$ \\
\hline Mild & 62 & 73.3 & $67.7-79.0$ & 84.4 & \\
\hline Moderate & 73 & 38.1 & $30.0-46.2$ & 37.9 & \\
\hline Marked & 25 & 34.5 & $21.3-47.8$ & 39.5 & \\
\hline TNM stage & & & & & \\
\hline I & 67 & 79.4 & $76.3-82.6$ & 93.9 & $<0.0001$ \\
\hline II & 37 & 70.1 & $61.8-78.4$ & 78.9 & \\
\hline III & 37 & 44.8 & $33.5-56.0$ & 47.6 & \\
\hline IV & 48 & 10.4 & $6.9-13.9$ & 0.0 & \\
\hline Claudin-4 & & & & & \\
\hline Negative & 29 & 64.2 & $53.0-75.3$ & 71.5 & 0.1690 \\
\hline Positive & 160 & 53.0 & $47.3-58.6$ & 58.0 & \\
\hline MMP-2 & & & & & \\
\hline Negative & 36 & 63.0 & $53.5-72.6$ & 71.5 & 0.1120 \\
\hline Positive & 153 & 52.4 & $46.6-58.2$ & 57.4 & \\
\hline
\end{tabular}


Table III. Continued

\begin{tabular}{lrcccc}
\hline Factors & Cases & Mean survival (months) & 95\% CI of mean & 5-year survival (\%) & P-value \\
\hline MMP-9 & & & & & \\
Negative & 20 & 68.3 & $56.0-80.7$ & 84.2 & 0.0410 \\
Positive & 169 & 52.9 & $47.5-58.3$ & 57.2 & \\
\hline
\end{tabular}

CI, confidence interval.

A

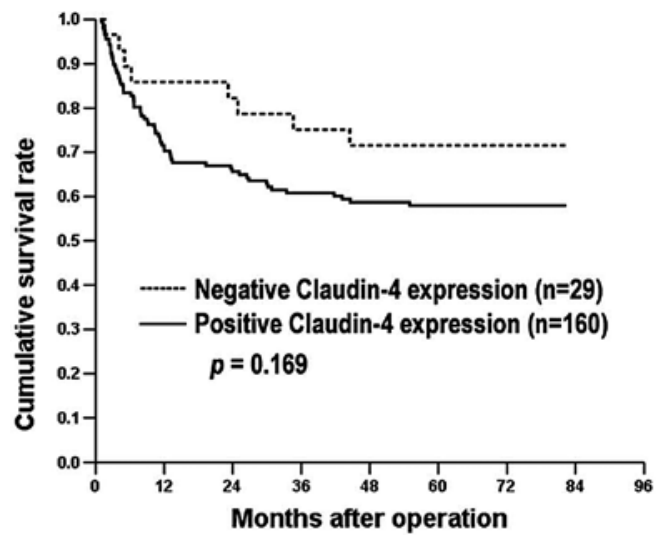

B

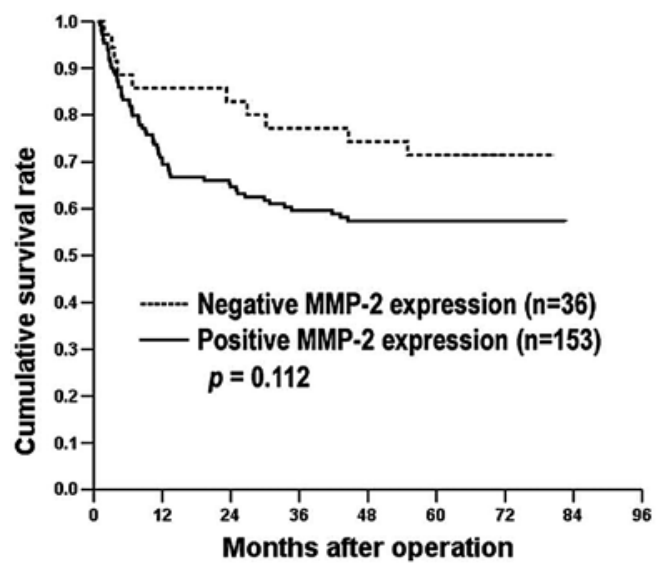

C

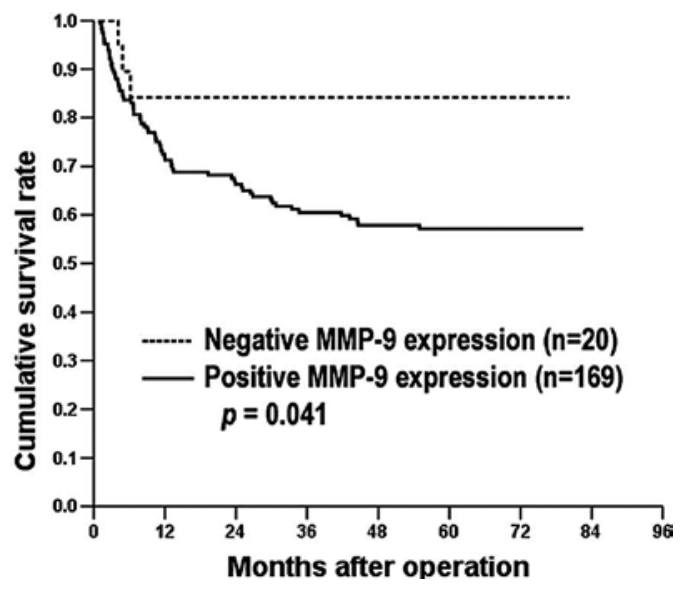

Figure 3. Kaplan-Meier survival curves for disease-free survival of 189 patients with gastric cancer. (A) Categorized by claudin-4 expression, no significant difference was observed between patients with positive claudin- 4 expression and those with negative $(\mathrm{P}=0.169)$. (B) Categorized by MMP-2 expression, no significant difference was observed between the two groups $(\mathrm{P}=0.112)$. (C) Categorized by MMP-9 expression, survival was significantly better for patients with negative MMP-9 expression than for those with positive $(\mathrm{P}=0.041)$.
Table IV. Multivariate Cox's proportional hazards analysis for disease-free survival of 189 gastric cancer patients undergoing gastrectomy.

\begin{tabular}{lrrr}
\hline Factors & \multicolumn{2}{c}{ Relative risk $(95 \% \mathrm{CI})$} & P-value \\
\hline Depth of invasion & & & $<0.0001$ \\
T2 vs. T1 & 5.543 & $(0.962-31.950)$ & 0.0550 \\
T3 vs.T1 & 20.420 & $(4.023-103.659)$ & 0.0003 \\
T4 vs. T1 & 35.392 & $(6.037-207.466)$ & $<0.0001$ \\
Lymph node metastasis & & & 0.0005 \\
N1 vs. N0 & 1.926 & $(0.831-4.465)$ & 0.1270 \\
N2 vs. N0 & 3.643 & $(1.516-8.756)$ & 0.0040 \\
N3 vs. N0 & 5.779 & $(2.387-13.990)$ & 0.0001 \\
Lymphatic invasion & & & \\
Yes vs. no & 2.115 & $(1.188-3.766)$ & 0.0110 \\
\hline
\end{tabular}

\section{References}

1. Tsukita $\mathrm{S}$ and Furuse $\mathrm{M}$ : Pores in the wall: claudins constitute tight junction strands containing aqueous pores. J Cell Biol 149: 13-16, 2000.

2. Tsukita $\mathrm{S}$ and Furuse M: Claudin-based barrier in simple and stratified cellular sheets. Curr Opin Cell Biol 14: 531-536, 2002.

3. Anderson JM: Molecular structure of tight junctions and their role in epithelial transport. News Physiol Sci 16: 126-130, 2001.

4. Cereijido M, Valdes J, Shoshani L, et al: Role of tight junctions in establishing and maintaining cell polarity. Annu Rev Physiol 60: 161-177, 1998.

5. Tsukita S, Furuse $M$ and Itoh M: Multifunctional strands in tight junctions. Nat Rev Mol Cell Biol 2: 285-293, 2001.

6. Morita K, Furuse M, Fujimoto K, et al: Claudin multigene family encoding four-transmembrane domain protein components of tight junction strands. Proc Natl Acad Sci USA 96: 511-516, 1999.

7. Itoh M, Furuse M, Morita $\mathrm{K}$, et al: Direct binding of three tight junction-associated MAGUKs, ZO-1, ZO-2 and ZO-3, with the COOH termini of claudins. J Cell Biol 147: 1351-1363, 1999.

8. Yamamoto Y, Nishimura N, Morimoto S, et al: Distinct roles of Rab3B and Rab13 in the polarized transport of apical, basolateral, and tight junctional membrane proteins to the plasma membrane. Biochem Biophys Res Commun 308: 270-275, 2003.

9. Balda MS, Garrett MD and Matter K: The ZO-1-associated Y-box factor ZONAB regulates epithelial cell proliferation and cell density. J Cell Biol 160: 423-432, 2003.

10. Wu Y, Dowbenko D, Spencer S, et al: Interaction of the tumor suppressor PTEN/MMAC with a PDZ domain of MAGI3, a novel membrane-associated guanylate kinase. J Biol Chem 275: 21477-21485, 2000.

11. Mullin JM: Potential interplay between luminal growth factors and increased tight junction permeability in epithelial carcinogenesis. J Exp Zool 279: 484-489, 1997.

12. Soler AP, Miller RD, Laughlin KV, et al: Increased tight junctional permeability is associated with the development of colon cancer. Carcinogenesis 20: 1425-1431, 1999. 
13. Resnick MB, Gavilanez M, Newton E, et al: Claudin expression in gastric adenocarcinomas: a tissue microarray study with prognostic correlation. Hum Pathol 36: 886-892, 2005.

14. Wu CM, Lee YS, Wang TH, et al: Identification of differential gene expression between intestinal and diffuse gastric cancer using cDNA microarray. Oncol Rep 15: 57-64, 2006.

15. Kuo WL, Lee LY, Wu CM, et al: Differential expression of claudin- 4 between intestinal and diffuse-type gastric cancer. Oncol Rep 16: 729-734, 2006.

16. Song X, Chen H, Shen B, et al: Expression of Cdx2 and claudin-2 in the multistage tissue of gastric carcinogenesis. Oncology 73 357-365, 2007.

17. Song X, Li X, Tang Y, et al: Expression of claudin-2 in the multistage process of gastric carcinogenesis. Histol Histopathol 23: $673-682,2008$

18. Long H, Crean CD, Lee WH, et al: Expression of Clostridium perfringens enterotoxin receptors claudin-3 and claudin-4 in prostate cancer epithelium. Cancer Res 61: 7878-7881, 2001.

19. Santin $\mathrm{AD}$, Zhan F, Cane S, et al: Gene expression fingerprint of uterine serous papillary carcinoma: identification of nove molecular markers for uterine serous cancer diagnosis and therapy. Br J Cancer 92: 1561-1573, 2005.

20. Nichols LS, Ashfaq R and Iacobuzio-Donahue CA: Claudin 4 protein expression in primary and metastatic pancreatic cancer: support for use as a therapeutic target. Am J Clin Pathol 121: 226-230, 2004

21. Al Moustafa AE, Alaoui-Jamali MA, Batist G, et al: Identification of genes associated with head and neck carcinogenesis by cDNA microarray comparison between matched primary normal epithelial and squamous carcinoma cells. Oncogene 21 2634-2640, 2002.

22. Kominsky SL, Argani P, Korz D, et al: Loss of the tight junction protein claudin-7 correlates with histological grade in both ductal carcinoma in situ and invasive ductal carcinoma of the breast. Oncogene 22: 2021-2033, 2003.

23. Kominsky SL, Vali M, Korz D, et al: Clostridium perfringens enterotoxin elicits rapid and specific cytolysis of breast carcinoma cells mediated through tight junction proteins claudin 3 and 4 . Am J Pathol 164: 1627-1633, 2004

24. Morson BC, Dawson IMP and Day DW: Morson and Dawson's Gastrointestinal Pathology. 3rd edition. Blackwell Science, Oxford, pp53-70, 1990.

25. Hart IR and Saini A: Biology of tumour metastasis. Lancet 339: 1453-1457, 1992

26. Kohn EC and Liotta LA: Molecular insights into cancer invasion: strategies for prevention and intervention. Cancer Res 55: 1856-1862, 1995

27. Murphy G and Docherty AJ: The matrix metalloproteinases and their inhibitors. Am J Respir Cell Mol Biol 7: 120-125, 1992.

28. Stetler-Stevenson WG, Liotta LA and Kleiner DE: Extracellular matrix 6: role of matrix metalloproteinases in tumor invasion and metastasis. FASEB J 7: 1434-1441, 1993.

29. Davies B, Waxman J, Wasan H, et al: Levels of matrix metalloproteases in bladder cancer correlate with tumor grade and invasion. Cancer Res 53: 5365-5369, 1993.

30. Boag AH and Young ID: Increased expression of the 72-kd type IV collagenase in prostatic adenocarcinoma. Demonstration by immunohistochemistry and in situ hybridization. Am J Patho 144: 585-591, 1994.

31. Muller D, Wolf C, Abecassis J, et al: Increased stromelysin 3 gene expression is associated with increased local invasiveness in head and neck squamous cell carcinomas. Cancer Res 53: $165-169,1993$

32. Urbanski SJ, Edwards DR, Hershfield N, et al: Expression pattern of metalloproteinases and their inhibitors changes with the progression of human sporadic colorectal neoplasia. Diagn Mol Pathol 2: 81-89, 1993.

33. Sakurai Y, Otani Y, Kameyama K, et al: Expression of interstitial collagenase (matrix metalloproteinase-1) in gastric cancers Jap J Cancer Res 88: 401-406, 1997.

34. Torii A, Kodera Y, Uesaka K, et al: Plasma concentration of matrix metalloproteinase 9 in gastric cancer. Br J Surg 84: 133-136, 1997.
35. Agarwal R, D'Souza T and Morin PJ: Claudin-3 and claudin-4 expression in ovarian epithelial cells enhances invasion and is associated with increased matrix metalloproteinase- 2 activity. Cancer Res 65: 7378-7385, 2005.

36. Oku N, Sasabe E, Ueta E, et al: Tight junction protein claudin-1 enhances the invasive activity of oral squamous cell carcinoma cells by promoting cleavage of laminin-5 gamma 2 chain via matrix metalloproteinase (MMP)-2 and membrane-type MMP-1. Cancer Res 66: 5251-5257, 2006.

37. Takehara M, Nishimura T, Mima S, et al: Effect of claudin expression on paracellular permeability, migration and invasion of colonic cancer cells. Biol Pharm Bull 32: 825-831, 2009.

38. Schraml P, Bucher C, Bissig H, et al: Cyclin E overexpression and amplification in human tumours. J Pathol 200: 375-382, 2003.

39. Ravn V, Havsteen $H$ and Thorpe SM: Immunohistochemical evaluation of estrogen and progesterone receptors in paraffinembedded, formalin-fixed endometrial tissues: comparison with enzyme immunoassay and immunohistochemical analysis of frozen tissue. Mod Pathol 11: 709-715, 1998.

40. Ravn V, Rasmussen BB and Hojholt L: Reproducibility of subjective immunohistochemical estrogen- and progesteronereceptor determination in human endometrium. Pathol Res Pract 189: 1015-1022, 1993

41. Wu YL, Zhang S, Wang GR, et al: Expression transformation of claudin-1 in the process of gastric adenocarcinoma invasion. World J Gastroenterol 14: 4943-4948, 2008.

42. Kinugasa T, Huo Q, Higashi D, et al: Selective up-regulation of claudin-1 and claudin-2 in colorectal cancer. Anticancer Res 27: 3729-3734, 2007

43. Dhawan P, Singh AB, Deane NG, et al: Claudin-1 regulates cellular transformation and metastatic behavior in colon cancer. J Clin Invest 115: 1765-1776, 2005.

44. Ueda J, Semba S, Chiba H, et al: Heterogeneous expression of claudin-4 in human colorectal cancer: decreased claudin-4 expression at the invasive front correlates cancer invasion and metastasis. Pathobiology 74: 32-41, 2007.

45. Oshima T, Kunisaki C, Yoshihara K, et al: Reduced expression of the claudin-7 gene correlates with venous invasion and liver metastasis in colorectal cancer. Oncol Rep 19: 953-959, 2008.

46. Usami Y, Chiba H, Nakayama F, et al: Reduced expression of claudin-7 correlats with invasion and metastasis in squamous cell carcinoma of the esophagus. Hum Pathol 37: 569-577, 2006.

47. Morohashi S, Kusumi T, Sato F, et al: Decreased expression of claudin-1 correlates with recurrence status in breast cancer. Int J Mol Med 20: 139-143, 2007.

48. Grigioni WF, D'Errico A, Fortunato C, et al: Prognosis of gastric carcinoma revealed by interactions between tumor cells and basement membrane. Mod Pathol 7: 220-225, 1994.

49. Tori A, Kodera Y, Ito M, et al: Matrix metalloproteinase 9 in mucosally invasive gastric cancer. Gastric Cancer 1: 142-145, 1998.

50. Schwartz GK: Invasion and metastasis in gastric cancer: in vitro and in vivo models with clinical correlations. Semin Oncol 23: 316-324, 1996

51. Kabashima A, Maehare Y, Kakeji Y, et al: Clinicopathological features and overexpression of matrix metalloproteinases in intramucosal gastric carcinoma with lymph node metastasis. Clin Cancer Res 6: 3581-3584, 2000.

52. Allgayer H, Babic R, Beyer BC, et al: Prognostic relevance of MMP-2 (72-kDa collagenase IV) in gastric cancer. Oncology 55: $152-160,1998$.

53. Ji F, Chen YL, Jin EY, et al: Relationship between matrix metalloproteinase-2 mRNA expression and clinicopathological and urokinase-type plasminogen activator system parameters and prognosis in human gastric cancer. World J Gastroenterol 11: 3222-3226, 2001

54. Monig SP, Baldus SE, Hennecken JK, et al: Expression of MMP-2 is associated with progression and lymph node metastasis of gastric carcinoma. Histopathology 39: 597-602, 2001. 